\title{
ON INTEGERS FREE OF LARGE PRIME FACTORS
}

\author{
ADOLF HILDEBRAND ${ }^{1}$ AND GÉRALD TENENBAUM
}

\begin{abstract}
The number $\Psi(x, y)$ of integers $\leq x$ and free of prime factors $>y$ has been given satisfactory estimates in the regions $y \leq(\log x)^{3 / 4-\varepsilon}$ and $y>\exp \left\{(\log \log x)^{5 / 3+\varepsilon}\right\}$. In the intermediate range, only very crude estimates have been obtained so far. We close this "gap" and give an expression which approximates $\Psi(x, y)$ uniformly for $x \geq y \geq 2$ within a factor $1+$ $O((\log y) /(\log x)+(\log y) / y)$. As an application, we derive a simple formula for $\Psi(c x, y) / \Psi(x, y)$, where $1 \leq c \leq y$. We also prove a short interval estimate for $\Psi(x, y)$.
\end{abstract}

1. Introduction. Let $\Psi(x, y)$ denote the number of positive integers not exceeding $x$ and free of prime factors larger than $y$. Besides their own interest, estimates for $\Psi(x, y)$ have found applications in various problems in number theory $[\mathbf{6 , 1 7}]$, and there exists a large literature on this subject. A comprehensive bibliography can be found in Norton's memoir [15].

The earliest result is due to Dickman [7] who gave, about fifty years ago, an asymptotic formula for $\Psi(x, y)$ in the case $x$ is a fixed power of $y$. Dickman showed that

$$
\Psi(x, y) \sim x \rho(u) \quad\left(x \rightarrow \infty, y=x^{1 / u}\right)
$$

for every fixed $u>0$, where $\rho(u)$, the "Dickman-function", is defined as the continuous solution of the differential difference equation

$$
u \rho^{\prime}(u)+\rho(u-1)=0 \quad(u>1),
$$

with the initial condition

$$
\rho(u)=1 \quad(0 \leq u \leq 1) .
$$

That a function defined in such a way occurs is a typical feature of a sieve problem. It is due to the fact that $\Psi(x, y)$ satisfies a functional equation, the Buchstab identity, which, after smoothing, reduces to the differential difference equation (1.2).

In 1938, in a famous paper on large differences of consecutive primes, Rankin [17] derived an upper bound for $\Psi(x, y)$ in a special case, namely when $\log x$ is of order $(\log y)^{2}$. To this end, he used a remarkably simple but effective device, which can be applied to many similar situations and has become known as "Rankin's method". It is based on the inequality

$$
\Psi(x, y) \leq \sum_{\substack{n \geq 1 \\ P(n) \leq y}}\left(\frac{x}{n}\right)^{\sigma}=x^{\sigma} \prod_{p \leq y}\left(1-p^{-\sigma}\right)^{-1} \quad(\sigma>0),
$$

Received by the editors June 24,1985 .

1980 Mathematics Subject Classification (1985 Revision). Primary 11N25.

${ }^{1}$ Supported by a grant from the Deutsche Forschungsgemeinschaft. 
where $P(n)$ stands for the largest prime factor of $n$. By choosing $\sigma$ optimally and estimating further the right-hand side of (1.4), one arrives at an upper bound for $\Psi(x, y)$ which turns out to be especially useful when $y$ is relatively small compared with $x$. When $y$ is a fixed or slowly decreasing power of $x$, however, (1.4) becomes weaker than the trivial bound $\Psi(x, y) \leq x$. Rankin's method was fully exploited by de Bruijn [4] in 1966.

Most of the work on $\Psi(x, y)$ in the literature deals with the case when $u=$ $(\log x) /(\log y)$ is relatively small. The aim is then to give quantitative and uniform versions of Dickman's relation (1.1). In 1951, de Bruijn [3] produced an estimate for $\Psi(x, y)$ which implies that the formula

$$
\Psi(x, y)=x \rho(u)\left(1+O_{\varepsilon}\left(\frac{\log (u+1)}{\log y}\right)\right)
$$

(where, here and in the sequel, we write systematically $u=(\log x) /(\log y)$ ), and hence (1.1), hold uniformly in the range

$$
x \geq 2, \quad \exp \left\{(\log x)^{5 / 8+\varepsilon}\right\}<y \leq x,
$$

where $\varepsilon$ is any fixed positive number. Similar results have been established subsequently by several authors on using different methods, but de Bruijn's estimate remained for a long time the best of its type. It can be shown that the error term in (1.5) is best possible, and it was only recently that the range of validity (1.6) was improved. Maier [14] established (1.5) for the range

$$
x \geq 2, \quad \exp \left\{(\log x)^{\varepsilon}\right\} \leq y \leq x .
$$

Hensley $[\mathbf{1 0}]$ proved the lower estimate

$$
\Psi(x, y) \gg_{\varepsilon} x \rho(u)
$$

for an even larger range, namely

$$
x \geq 2, \quad \exp \left\{(\log \log x)^{5 / 3+\varepsilon}\right\}<y \leq x .
$$

In [12] the first author showed that de Bruijn's formula (1.5) actually holds in the above range (1.7). It seems that this is the limit of what can be reached unconditionally. On the Riemann hypothesis, one can prove that (1.5) holds in the range $x \geq 2,(\log x)^{2+\varepsilon}<y \leq x$.

The behavior of $\Psi(x, y)$ is quite different when $y$ is very small compared with $x$. Ennola [9] showed that uniformly for

$$
2 \leq y \leq \sqrt{\log x}
$$

the estimate

$$
\Psi(x, y)=\frac{1}{\pi(y) !} \prod_{p \leq y}\left(\frac{\log x}{\log p}\right)\left(1+O\left(\frac{y^{2}}{\log x \log y}\right)\right)
$$

holds, where $\pi(y)$ is the prime counting function. The right-hand side of (1.9) depends strongly on irregularities in the distribution of primes and cannot be replaced by a smooth function as in (1.5). Ennola also gave a similar, but much more complicated, formula for the range

$$
2 \leq y \leq(\log x)^{3 / 4-\varepsilon} .
$$


Between the ranges (1.10) and (1.7), in which asymptotic formulae for $\Psi(x, y)$ are known, there remains a large gap where the estimates available are much less precise. For these intermediate values, the best known upper bound is due to de Bruijn [4] and achieved by Rankin's method. Various lower bounds have been given $[4,6,10,12]$, but they are far from being asymptotically equal to the corresponding upper estimates. In the case $y=\log x$, for instance, the known upper and lower bounds differ by a factor $\gg_{\varepsilon} \exp \left\{y^{1-\varepsilon}\right\}$ for every $\varepsilon>0$.

The main purpose of this paper is to close this gap and give an expression approximating $\Psi(x, y)$ uniformly for $x \geq y \geq 2$ within a factor $1+O(1 / u+(\log y) / y)$ and thus yield an asymptotic formula whenever $y$ and $u=(\log x) /(\log y)$ tend to infinity.

2. Statement of results. For complex $s=\sigma+i \tau$, put

$$
\varsigma(s, y)=\prod_{p \leq y}\left(1-p^{-s}\right)^{-1} .
$$

This finite part of the Euler product for the Riemann zeta function is meromorphic in $s$ in the whole plane. It never vanishes, and all its poles are located on the line $\sigma=0$. We also set

$$
\phi(x, y):=\log \varsigma(s, y) \quad(\sigma>0)
$$

and denote by $\phi_{k}(s, y), k \geq 0$, the $k$ th partial derivative of $\phi(s, y)$ with respect to $s$. From the definition of $\phi(s, y)$ we thus have

$$
\phi_{1}(s, y)=-\sum_{p \leq y} \frac{\log p}{p^{s}-1}, \quad \phi_{2}(s, y)=\sum_{p \leq y} \frac{p^{s}(\log p)^{2}}{\left(p^{s}-1\right)^{2}} .
$$

By Rankin's upper bound (1.4) we have

$$
\Psi(x, y) \leq \inf _{\sigma>0} x^{\sigma} \zeta(\sigma, y)
$$

This infimum is in fact a minimum, and is attained for $\sigma=\alpha=\alpha(x, y)$ defined as the (unique) solution of the equation

$$
\phi_{1}(\alpha, y)+\log x=0 .
$$

We reserve henceforth the letter $\alpha$ to denote the solution of (2.2). Our approximation for $\Psi(x, y)$ will consist of the right-hand side of $(2.1)$, namely $x^{\alpha} \zeta(\alpha, y)$, multiplied by a factor of a fairly simple shape.

We now state our main result.

THEOREM 1. We have uniformly for $x \geq y \geq 2$,

$$
\Psi(x, y)=\frac{x^{\alpha} \zeta(\alpha, y)}{\alpha \sqrt{2 \pi \phi_{2}(\alpha, y)}}\left(1+O\left(\frac{1}{u}+\frac{\log y}{y}\right)\right) .
$$

It is plain from the definitions that $\alpha$, and hence $\phi_{2}(\alpha, y)$, are positive. Thus dividing the right-hand side of $(2.1)$ by $\alpha \sqrt{2 \pi \phi_{2}(\alpha, y)}$ yields an asymptotic formula for $\Psi(x, y)$ whenever $y$ and $u$ tend to infinity.

For applications, it is useful to have approximations of $\alpha=\alpha(x, y)$ and $\phi_{2}(\alpha, y)$ at hand. Although these quantities are defined only implicitly, via prime number sums, they can be replaced in (2.3) by smooth functions at the cost of a weaker error 
term. The following theorem shows that they can both be well approximated over the whole range $x \geq y \geq 2$. This takes into account the factor $\left(\alpha \sqrt{2 \pi \phi_{2}(\alpha, y)}\right)^{-1}$ in (2.3) and enables one to compare Rankin's upper bound with the true order of magnitude of $\Psi(x, y)$. Moreover, if $x$ is not too large with respect to $y$, we can derive a reasonably smooth approximation for the whole right-hand side of (2.3).

THEOREM 2. (i) We have uniformly for $x \geq y \geq 2$,

$$
\alpha(x, y)=\frac{\log (1+y / \log x)}{\log y}\left(1+O\left(\frac{\log \log (1+y)}{\log y}\right)\right)
$$

and

$$
\phi_{2}(\alpha, y)=\left(1+\frac{\log x}{y}\right) \log x \cdot \log y\left(1+O\left(\frac{1}{\log (1+u)}+\frac{1}{\log y}\right)\right) .
$$

(ii) Let $\varepsilon, 0<\varepsilon \leq 1 / 2$, be fixed. Then we have uniformly for $x \geq 2$, $(\log x)^{1+\varepsilon}<$ $y \leq x$,

$$
\begin{aligned}
& \frac{x^{\alpha} \zeta(\alpha, y)}{\alpha \sqrt{2 \pi \phi_{2}(\alpha, y)}} \\
& =x\left(\frac{\xi^{\prime}(u)}{2 \pi}\right)^{1 / 2} \exp \left\{\gamma-u \xi(u)+\int_{0}^{\xi(u)} \frac{e^{s}-1}{s} d s\right. \\
& \left.+O_{\varepsilon}\left(\frac{\log (1+u)}{\log y}+u \exp \left\{-(\log y)^{3 / 5-\varepsilon}\right\}\right)\right\},
\end{aligned}
$$

where $\gamma$ denotes Euler's constant, $\xi=\xi(u)$ is the unique positive solution of the equation $e^{\xi}=1+u \xi$ and $\xi^{\prime}(u)$ is the derivative of the function $\xi(u)$.

From part (i) of Theorem 2, we see that

$$
\alpha \sqrt{2 \pi \phi_{2}(\alpha, y)} \ll \sqrt{y / \log y} .
$$

Thus Rankin's upper bound differs from $\Psi(x, y)$ by a factor at most $O(\sqrt{y / \log y})$. This is surprisingly small, compared with the precision of the estimates for $\Psi(x, y)$ available in the literature, which in the case $y=\log x$, for example, involve an error factor of order $\gg_{\varepsilon} \exp \left\{y^{1-\varepsilon}\right\}$. On the other hand, (2.4) and (2.5) also imply

$$
\alpha \sqrt{2 \pi \phi_{2}(\alpha, y)} \gg \log y
$$

uniformly for $x \geq y \geq 2$, so we never have asymptotic equality in (2.1).

Combining Theorem 1 with part (i) of Theorem 2, we obtain the following corollary.

COROLlARY 1. If $y$ and $u$ tend to infinity, we have

$$
\Psi(x, y) \sim x^{\alpha} \zeta(\alpha, y)\left(2 \pi u\left(1+\frac{\log x}{y}\right)\right)^{-1 / 2}\left(\log \left(1+\frac{y}{\log x}\right)\right)^{-1} .
$$

In particular

$$
\Psi(x, y) \sim \frac{x^{\alpha} \varsigma(\alpha, y)}{\sqrt{2 \pi u} \log (y / \log x)} \quad(\text { if } y / \log x \rightarrow \infty)
$$


and

$$
\Psi(x, y) \sim \frac{x^{\alpha} \zeta(\alpha, y)}{\sqrt{2 \pi y / \log y}} \quad(\text { if } y / \log x \rightarrow 0) .
$$

This shows that the behaviour of $\Psi(x, y)$ is quite different according as $y / \log x$ is small or large. This phenomenon has already been stressed by de Bruijn [4]. It is due to the fact that for $y \geq y_{0}(\varepsilon)$ and $k y \leq(1-\varepsilon) \log x$, we have by the prime number theorem that $\left(\prod_{p \leq y} p\right)^{k} \leq x$ so that the numbers that do not have a prime factorization with exceptionally high powers contribute little to $\Psi(x, y)$-for instance the squarefree numbers contribute at most $x^{1 / k}$. This feature does not occur when $y / \log x$ is large.

When we combine Theorem 1 with part (ii) of Theorem 2, we derive a smooth approximation for $\Psi(x, y)$, which in the range

$$
x \geq 2, \quad(\log \log x)^{5 / 3+\varepsilon}<\log y \leq \sqrt{\log x}
$$

is as sharp as de Bruijn's estimate (1.5). Apart from the upper restriction for $y$, this range coincides with (1.7), where (1.5) is known to be valid. For larger $y$, estimates (2.3) and (2.6) together are less precise than (1.5) because of the error term $O(1 / u)$ in $(2.3)$.

The approximations (1.5) and (2.6) are of quite different type. Equalizing the two expressions in the case $u=(\log 2 y)^{1 / 2}$, say, we obtain the following result.

Corollary 2. As $u \rightarrow \infty$, we have

$$
\rho(u)=\left(1+O\left(\frac{1}{u}\right)\right)\left(\frac{\xi^{\prime}(u)}{2 \pi}\right)^{1 / 2} \exp \left\{\gamma-u \xi(u)+\int_{0}^{\xi(u)} \frac{e^{s}-1}{s} d s\right\} .
$$

This formula is not new. Thirty years ago, de Bruijn [2] established the asymptotic relation

$$
\rho(u) \sim \frac{1}{\sqrt{2 \pi u}} \exp \left\{\gamma-u \xi(u)+\int_{0}^{\xi(u)} \frac{e^{s}-1}{s} d s\right\},
$$

which is a weaker form of (2.7) since $\xi^{\prime}(u) \sim 1 / u$, as can easily be shown. De Bruijn's proof was quite elaborate. A different demonstration has been given recently by Canfield [5]. The more precise formula (2.7) has been first proved by Alladi [1, p. 186] using de Bruijn's method. In our case, this formula arises quite naturally, since both sides appear as approximations, obtained from different methods, for the same arithmetical quantity $\Psi(x, y) / x$.

From Theorem 1, we shall deduce the following simple formula for the ratio $\Psi(c x, y) / \Psi(x, y)$, where $1 \leq c \leq y$.

THEOREM 3. We have uniformly for $x \geq y \geq 2$ and $1 \leq c \leq y$,

$$
\Psi(c x, y)=\Psi(x, y) c^{\alpha(x, y)}\left(1+O\left(\frac{1}{u}+\frac{\log y}{y}\right)\right) .
$$

This improves on results of Hensley [11] and the first author [13], where estimates of the same type, but with weaker error terms and only for a restricted range, had been obtained. 
The factor $c^{\alpha(x, y)}$ can be well approximated by a smooth function. For instance, it follows from part (i) of Theorem 2 that the estimate

$$
c^{\alpha(x, y)}=\left(1+\frac{y}{\log x}\right)^{(\log c) /(\log y)}\left(1+O\left(\frac{\log \log (1+y)}{\log y}\right)\right)
$$

holds uniformly for $x \geq y \geq 2$ and $1 \leq c \leq 2$. Substituting in Theorem 3 we derive as an immediate consequence the following result.

COROLlary 3. Let $x$ and $y$ tend to infinity.

Then the relation $\Psi(2 x, y) \sim \Psi(x, y)$ holds if and only if $\log y \leq(1+o(1)) \log \log x$. Moreover, we have $\Psi(2 x, y) \sim 2 \Psi(x, y)$ if and only if $(\log y) /(\log \log x) \rightarrow \infty$.

The first of these asymptotic formulae is new; the second has been recently proved by Hensley $[\mathbf{1 1}]$, using a completely different method. The corollary shows once more how the behaviour of $\Psi(x, y)$ changes at $y \sim \log x$.

Theorem 3 can also be interpreted as a short interval estimate for $\Psi(x, y)$. It leads to an asymptotic formula for $\Psi(x+x / z, y)-\Psi(x, y)$ in terms of $\Psi(x, y)$ provided $z$ is not too large. More precisely, $z$ has to be smaller than $u$ and $y / \log y$. Setting, for $0<\varepsilon<1$,

$$
Y(\varepsilon)=\exp \left\{(\log y)^{3 / 2-\varepsilon}\right\}
$$

we can in fact sharpen Theorem 3 for large values of $z$ in the following way.

THEOREM 4. We have uniformly for $x \geq y \geq 2, z \geq 1,0<\varepsilon<1$,

$$
\begin{aligned}
\Psi\left(x+\frac{x}{z}, y\right)-\Psi(x, y)= & \frac{\alpha(x, y)}{z} \Psi(x, y)\left(1+O\left(\frac{1}{z}+\frac{1}{u}+\frac{\log y}{y}\right)\right) \\
& +O_{\varepsilon}(\Psi(x, y) R(x, y))
\end{aligned}
$$

with

$$
R(x, y):=Y(\varepsilon)^{-1}+\exp \left\{-b_{0} u(\log 2 u)^{-2}\right\}(\log y)
$$

for some positive absolute constant $b_{0}$.

The last error term in (2.10) can be omitted if we suppose $\alpha / z \gg R(x, y)^{1 / 2}$ and modify adequately $b_{0}$ and $\varepsilon$. Using estimate $(2.4)$ for $\alpha(x, y)$, we see that this condition is certainly fulfilled in the range

$$
\left\{\begin{array}{l}
x \geq 2, \\
1 \leq z \leq Y(\varepsilon) .
\end{array} \quad(\log \log x)^{2 / 3+\varepsilon}<\log y \leq(\log x)^{2 / 5},\right.
$$

Thus, we can state the following corollary.

COROLlary 4. Let $\varepsilon, 0<\varepsilon<1$, be fixed. Then we have uniformly in the range (2.11)

$$
\Psi\left(x+\frac{x}{z}, y\right)-\Psi(x, y)=\frac{\log (1+y / \log x)}{z \log y} \Psi(x, y)\left(1+O_{\varepsilon}\left(\frac{1}{z}+\frac{\log \log (1+y)}{\log y}\right)\right) .
$$

Under the Riemann Hypothesis, Theorem 4 is valid with $Y(\varepsilon)=\exp \left\{y^{1 / 2-\varepsilon}\right\}$. Corollary 4 then holds as stated if $Y(\varepsilon)$ is replaced by $R(x, y)^{-1}$ in $(2.11)$.

Corollary 4 should be compared with Theorem 3 of $[\mathbf{1 2}]$, where the relation

$$
\Psi\left(x+\frac{x}{z}, y\right)-\Psi(x, y)=\frac{x \rho(u)}{z}\left(1+O_{\varepsilon}\left(\frac{\log (1+u)}{\log y}\right)\right)
$$


had been proved for the range (1.7) and $1 \leq z \leq y^{5 / 12}$. Since (1.5) holds in the same range, the term $x \rho(u)$ in (2.13) can be replaced by $\Psi(x, y)$, and we get an estimate of the same type as (2.12) with a slightly better error term. Hence, (2.12) can be regarded as an extension of (2.13) to a much larger range.

To prove Theorems 1 and 4, we use a somewhat "naive" analytic approach, relating $\Psi(x, y)$ to its Dirichlet series $\zeta(s, y)$ by a standard Perron-type formula; viz.,

$$
\Psi(x, y)=\frac{1}{2 \pi i} \int_{\kappa-i T}^{\kappa+i T} \varsigma(s, y) \frac{x^{s}}{s} d s+\text { Error } \quad(\kappa>0) .
$$

Such a method cannot reasonably succeed unless the free parameter $\kappa$ is chosen optimally. We select $\kappa=\alpha(x, y)$, which, by definition, is a saddle point for the function $\zeta(s, y) x^{s}$. The main contribution to the integral in (2.14) will then come from a small neighbourhood of the real point $s=\kappa=\alpha$, and integration over this range introduces the factor $\left(2 \pi \phi_{2}(\alpha, y)\right)^{-1 / 2}$, which is characteristic in the saddle point method.

The choice $\kappa=\alpha$ seems to be an essential feature here. Formulae like (2.14) have already been applied by several authors $[3,9,18]$ but without using the saddle point method in the process of estimating the complex integral. The results were not very satisfactory.

The above method can be applied in very general circumstances. For example, one can derive analogous estimates for the quantity $B_{l}(k ; x, y)$, defined as the number of positive integers $\leq x$ which are free of prime factors $>y$ and congruent to $l$ modulo $k$. This quantity already appears in the literature; see e.g. [15]. For fixed $k, l$ our method carries over smoothly; difficulties arise only when uniform estimates are required.

Another way of generalizing the above results is to consider the quantity

$$
\Psi_{f}(x, y)=\sum_{\substack{n \leq x \\ P(n) \leq y}} f(n)
$$

where $f$ is a multiplicative function of modulus at most 1 , say. Of special interest are the cases when $f$ is the Moebius function or is of the form $\exp \{i g(n)\}$, where $g$ is a real-valued additive function. In the latter situation, estimates for $\Psi_{f}(x, y)$ can be used to study the distribution of the additive function $g$ over the set of integers counted in $\Psi(x, y)$. This topic will be dealt with in another paper.

3. Notations and lemmas. For the reader's convenience, we recall here the main notations that will be used systematically in the sequel.

The letter $p$ always denotes a prime number and, for any integer $n$, we write $P(n)$ for the largest prime factor of $n$. By convention, $P(1)=1$.

The letter $s$ stands for a complex variable, defining implicitly $\sigma$ and $\tau$ by $s=$ $\sigma+i \tau$.

We put for $\sigma>0, y \geq 2$,

$$
\varsigma(s, y)=\prod_{p \leq y}\left(1-p^{-s}\right)^{-1}, \quad \phi(s, y)=\log \varsigma(x, y),
$$

and

$$
\phi_{k}(s, y)=\frac{\partial^{k}}{\partial s^{k}} \phi(s, y) \quad(k \geq 0)
$$


We write systematically for $x \geq y \geq 2,0<\varepsilon<1$,

$$
\begin{gathered}
Y(\varepsilon)=\exp \left((\log y)^{3 / 2-\varepsilon}\right), \\
u=\frac{\log x}{\log y}, \quad \bar{u}=\min \left(\frac{y}{\log y}, u\right)=\frac{\min (y, \log x)}{\log y}
\end{gathered}
$$

and let $\alpha=\alpha(x, y)$ be defined by the equation

$$
\left(-\phi_{1}(\alpha, y)=\right) \sum_{p \leq y} \frac{\log p}{p^{\alpha}-1}=\log x
$$

For $u>1$ we define $\xi=\xi(u)$ as the nonzero solution of the equation

$$
e^{\xi}=1+u \xi
$$

and put $\xi(1)=0$.

Finally, we let $c_{0}, c_{1}, \ldots$ denote positive absolute constants.

LEMMA 1. We have $u \geq 3$,

$$
\xi(u)=\log (u \log u)+O\left(\frac{\log \log u}{\log u}\right) .
$$

PROOF. By iteration of equation (3.2) we may write

$$
\begin{aligned}
\xi & =\log u+\log (\xi+1 / u)=\log u+\log (\log u+\log (\xi+1 / u)+1 / u) \\
& =\log (u \log u)+O(\log (\xi+1 / u) / \log u) .
\end{aligned}
$$

This implies the conclusion since we have trivially $1 \ll \xi \ll \log u$.

LEMMA 2. For $x \geq y \geq 2$, we have

$$
\begin{gathered}
\alpha(x, y) \asymp \frac{y}{u(\log y)^{2}} \quad(y \leq \log x), \\
\alpha(x, y) \geq \frac{1}{6 \log y} \quad(y>\log x), \\
(1-\alpha(x, y)) \log y=\xi(u)+O\left(\frac{1}{u}+\frac{\log x}{y}+e^{-\sqrt{\log y}}\right) \quad(y>\log x) .
\end{gathered}
$$

PROOF. The first two estimations are easily proved. Indeed, we have for $x \geq$ $y \geq 2$,

$$
u \log y=\sum_{p \leq y} \frac{\log p}{p^{\alpha}-1} \geq \frac{\theta(y)}{y^{\alpha}-1} \geq \frac{y / 5}{y^{\alpha}-1},
$$

where $\theta$ is the usual Tchebycheff function, and

$$
u \log y=\sum_{p \leq y} \frac{\log p}{p^{\alpha}-1} \leq \sum_{p \leq y} \frac{1}{\alpha} \ll \frac{y}{\alpha \log y} .
$$

This last estimate gives the upper bound of (3.3). By (3.6), we may write

$$
\alpha \geq \frac{\log (1+y /(5 u \log y))}{\log y}
$$

which implies the lower bounds of (3.3) and (3.4).

Formula (3.5) has been established in [13, Lemma 4] for $y \geq 2 \log x$. The proof is in fact valid in the case considered here. 
LEMMA 3. We have uniformly for $x \geq y \geq 2$,

$$
\left(y^{1-\alpha}-1\right) /((1-\alpha) \log y) \asymp \bar{u} .
$$

PROOF. We may plainly suppose that $y \geq y_{0}$, where $y_{0}$ is a sufficiently large constant.

First, consider the case $y \leq \log x$. From (3.3) we infer

$$
\alpha(x, y) \leq c_{1} / \log y<1 / 2 .
$$

This implies (3.7) since we also have $\bar{u}=y / \log y$.

If $y>\log x$, we use (3.5) in the form

$$
(1-\alpha) \log y=\xi(u)+O(1) .
$$

This gives the conclusion if $u \leq u_{0}$, say. If $u>u_{0}$, we may suppose by Lemma 1 that $\xi(u)$ is large, whence

$$
\frac{y^{1-\alpha}-1}{(1-\alpha) \log y}=\frac{e^{\xi+O(1)}}{\xi+O(1)} \asymp \frac{e^{\xi}}{\xi} .
$$

Now, by (3.2) and Lemma 1 ,

$$
e^{\xi} / \xi=u(1+1 / u \xi)=u \exp \{O(1 /(u \log u))\}
$$

and (3.7) follows.

LEMMA 4. We have uniformly for $x \geq y \geq 2$ and $1 \leq k \leq 4$,

$$
O<(-1)^{k} \phi_{k}(\alpha, y) \asymp(u \log y)^{k}(\bar{u})^{1-k} .
$$

ProOF. Differentiating $\phi(s, y)$ yields

$$
(-1)^{k} \phi_{k}(\alpha, y)=\sum_{p \leq y} \frac{(\log p) Q_{k-1}\left(p^{\alpha} \log p\right)}{\left(p^{\alpha}-1\right)^{k}}
$$

where $Q_{k-1}$ is a polynomial of degree $k-1$ with nonnegative coefficients.

If $y \leq \log x$, we have $\alpha \ll 1 / \log y$ by (3.3), whence

$$
(-1)^{k} \phi_{k}(\alpha, y) \asymp \sum_{p \leq y} \frac{(\log p)^{k}}{(\alpha \log p)^{k}} \asymp \frac{y}{\alpha^{k} \log y}=\bar{u} \alpha^{-k}
$$

and the desired estimate follows from (3.3).

If $y>\log x$, then $\alpha \gg 1 / \log y$ by (3.4) and

$$
\begin{aligned}
\frac{Q_{k-1}\left(p^{\alpha} \log p\right)}{\left(p^{\alpha}-1\right)^{k-1}} & \ll(\log p)^{k-1}\left(\frac{p^{\alpha}}{p^{\alpha}-1}\right)^{k-1} \\
& \ll(\log p)^{k-1}\left(1+\frac{1}{\alpha \log p}\right)^{k-1} \ll(\log y)^{k-1}
\end{aligned}
$$

Therefore we have

$$
(-1)^{k} \phi_{k}(\alpha, y) \ll(\log y)^{k-1} \sum_{p \leq y} \frac{\log p}{p^{\alpha}-1}=u(\log y)^{k}
$$


which is the upper bound wanted. For the lower bound, we use $\alpha \gg 1 / \log y$ in the form

$$
\frac{Q_{k-1}\left(p^{\alpha} \log p\right)}{\left(p^{\alpha}-1\right)^{k-1}} \gg(\log y)^{k-1} \quad(\sqrt{y}<p \leq y),
$$

whence

$$
(-1)^{k} \phi_{k}(\alpha, y) \gg(\log y)^{k-1} \sum_{\sqrt{y}<p \leq y} \frac{\log p}{p^{\alpha}} .
$$

The $p$-sum is easily seen to be $\gg\left(y^{1-\alpha}-1\right) /(1-\alpha)$ by Tchebycheff's estimates for the $\theta$-function. The result then follows by Lemma 3 .

LEMMA 5. We have uniformly for $x \geq y \geq 2$,

$$
S:=\frac{1}{\log y} \sum_{\nu \geq 2} \sum_{p^{\nu} \leq y} p^{-\nu \alpha} \log p \ll 1+y^{1 / 2-\alpha} .
$$

ProOF. Plainly

$$
S \ll \frac{1}{\log y} \sum_{p \leq \sqrt{y}} \frac{\log p}{p^{2 \alpha}} \quad\left(\text { if } \alpha \geq \frac{1}{4}\right)
$$

and

$$
S \ll \frac{1}{\log y} \sum_{p \leq \sqrt{y}} \frac{\log p}{p^{2 \alpha}}\left[\frac{\log y}{\log p}\right] \quad\left(\text { if } \alpha<\frac{1}{4}\right),
$$

In both cases the right-hand side is $\ll 1+y^{1 / 2-\alpha}$, by partial summation.

LEMMA 6. Let $y \geq 2,0<\beta, \varepsilon<1, \tau \in \mathbf{R},|\tau| \leq Y(\varepsilon), s=1-\beta+i \tau$. Then we have

$$
\sum_{n \leq y} \Lambda(n) n^{-s}=\frac{y^{\beta-i \tau}}{\beta-i \tau}+O_{\varepsilon}\left(\frac{1}{\beta}\left(1+y^{\beta} \exp \left\{-(\log y)^{\varepsilon / 2}\right\}\right)\right) .
$$

This result may be proved by usual contour integration starting with Perron's formula (see e.g. [16, Satz A.31]) and using Vinogradov's zero-free region for $\varsigma(s)$ (see e.g. [8, Theorem 11.2]). We omit the details.

COROLlaRY. On the hypotheses of Lemma 6, put

$$
\delta=\tau \log y-\operatorname{Arctan}(\tau / \beta)
$$

Then we have

$$
\begin{aligned}
& \sum_{n \leq y} \Lambda(n) n^{\beta-1}(1-\cos (\tau \log n)) \\
& \quad=\frac{y^{\beta}}{\beta}\left(1-\beta \frac{\cos \delta}{\sqrt{\beta^{2}+\tau^{2}}}\right)+O_{\varepsilon}\left(\frac{1}{\beta}\left(1+y^{\beta} \exp \left\{-(\log y)^{\varepsilon / 2}\right\}\right)\right) .
\end{aligned}
$$

PROOF. It suffices to apply (3.10) with $s=1-\beta$ and $s=1-\beta+i \tau$ and to take the real part of the difference.

The next lemma gathers a few simple inequalities that we shall need later and the proof of which we leave to the reader. 
LEMMA 7. We have

$$
\begin{array}{cc}
2 \theta^{2} / \pi^{2} \leq 1-\cos \theta \leq \theta^{2} / 2 & (|\theta| \leq \pi), \\
e^{v}\left(1-e^{-v}\right)^{2} \geq v^{2} \quad(v \in \mathbf{R}), \\
(1+t)^{-1} \leq \exp \{-t /(1+\lambda)\} & (0 \leq t \leq \lambda), \\
\left(1+4 v t /(t-1)^{2}\right)^{-1} \leq \exp \{-4 v / t\} & (0 \leq v \leq 1<t) .
\end{array}
$$

We can now prove the following result, which constitutes the main tool in the process of majorizing the contribution of large $\tau$ in the Perron integral for $\Psi(x, y)$.

LEMMA 8. Let $x \geq y \geq 2, \tau \in \mathbf{R}, s=\alpha(x, y)+i \tau$.

(i) If $|\tau| \leq 1 / \log y$, then

$$
\left|\frac{\varsigma(s, y)}{\varsigma(\alpha, y)}\right| \leq \exp \left\{-\frac{c_{0} y}{\log y} \log \left(1+\frac{\tau^{2} \phi_{2}(\alpha, y)}{(y / \log y)}\right)\right\} \text {. }
$$

(ii) For fixed $\varepsilon>0$, the estimate

$$
\left|\frac{\zeta(s, y)}{\zeta(\alpha, y)}\right| \ll_{\varepsilon} \exp \left\{-c_{0} \frac{\bar{u} \tau^{2}}{(1-\alpha)^{2}+\tau^{2}}\right\}
$$

holds uniformly for

$$
y \geq y_{0}(\varepsilon), \quad 1 / \log y<|\tau| \leq Y(\varepsilon),
$$

where $y_{0}(\varepsilon)$ is a sufficiently large absolute constant.

Proof. By an easy computation, we have

$$
\left|\frac{1-p^{-\alpha}}{1-p^{-s}}\right|=\left(1+\frac{2(1-\cos (\tau \log p))}{p^{\alpha}\left(1-p^{-\alpha}\right)^{2}}\right)^{-1 / 2} \text {. }
$$

We first consider the case $|\tau| \leq 1 / \log y$. If $y \leq \log x$, we have by (3.17) and (3.11), for $p \leq y$,

$$
\begin{aligned}
\left|\frac{1-p^{-\alpha}}{1-p^{-s}}\right| & \leq \exp \left\{-\frac{1}{2} \log \left(1+\frac{4 \tau^{2}(\log p)^{2} / \pi^{2}}{p^{\alpha}\left(1-p^{-\alpha}\right)^{2}}\right)\right\} \\
& \leq \exp \left\{-\frac{2}{\pi^{2}} \log \left(1+\tau^{2} \alpha^{-2} y^{-\alpha}\right)\right\} .
\end{aligned}
$$

Taking the product over $p \leq y$ yields (3.15) since by (3.3) and (3.8),

$$
y^{\alpha} \ll 1 \text { and } \alpha^{-2} \asymp u^{2} y^{-2}(\log y)^{4} \asymp \frac{\phi_{2}(\alpha, y)}{y / \log y} .
$$

If $y>\log x$, then $\alpha \gg 1 / \log y$ by (3.4), whence by (3.11) and (3.12),

$$
\frac{2(1-\cos (\tau \log p))}{p^{\alpha}\left(1-p^{-\alpha}\right)^{2}} \leq\left(\frac{\tau \log p}{\alpha \log p}\right)^{2} \leq c_{1}, \quad \text { say. }
$$

Next, we apply (3.13) with $\lambda=c_{1}$ to the right-hand side of (3.17). We obtain

$$
\left|\frac{1-p^{-\alpha}}{1-p^{-s}}\right| \leq \exp \left\{-\frac{1-\cos (\tau \log p)}{(1+\lambda) p^{\alpha}\left(1-p^{-\alpha}\right)^{2}}\right\} \leq \exp \left\{-c_{2} p^{\alpha}\left(\frac{\tau \log p}{p^{\alpha}-1}\right)^{2}\right\}
$$


with $c_{2}=2 /\left(\pi^{2}(1+\lambda)\right)$. Multiplying this for $p \leq y$ gives again (3.15), since in the ranges considered

$$
\tau^{2} \phi_{2}(\alpha, y) \ll u \ll y / \log y
$$

We now turn our attention to the case $|\tau|>1 / \log y$. It suffices to consider the case $u \geq u_{0}(\varepsilon)$, the result being trivial otherwise. We suppose that $u_{0}$ is so large that

$$
y^{1-\alpha} \asymp \bar{u} \log \bar{u} \quad\left(y \geq y_{0}(\varepsilon), u \geq u_{0}(\varepsilon)\right) .
$$

By Lemma 3, this is possible.

Now, by (3.17) and (3.14) with $v=(1-\cos (\tau \log p)) / 2, t=p^{\alpha}$,

$$
\left|\frac{1-p^{-\alpha}}{1-p^{-s}}\right| \leq \exp \left\{-(1-\cos (\tau \log p)) p^{-\alpha}\right\}
$$

whence $|\zeta(s, y) / \varsigma(\alpha, y)| \leq e^{-W}$ with

$$
W:=\sum_{p \leq y}(1-\cos (\tau \log p)) p^{-\alpha}
$$

Thus, we need a lower bound for $W$. We have trivially

$$
\frac{1}{\log y} \sum_{n \leq y} \Lambda(n)(1-\cos (\tau \log n)) n^{-\alpha} \leq W+2 S,
$$

where $S$ is the quantity introduced in Lemma 5. Applying the estimate of this lemma and corollary to Lemma 6 with $\beta=1-\alpha(x, y)$ yields, if $y \leq \log x$,

$$
\begin{aligned}
W & \gg \frac{y}{\log y}\left(1-\frac{1}{\sqrt{1+\tau^{2}}}+O_{\varepsilon}\left(\exp \left\{-(\log y)^{\varepsilon / 2}\right\}\right)\right)-c_{3} y^{1 / 2} \\
& \gg \frac{y \tau^{2}}{(\log y)\left(1+\tau^{2}\right)}
\end{aligned}
$$

and, if $y>\log x$,

$$
\begin{aligned}
W & \geq \frac{y^{1-\alpha}}{(1-\alpha) \log y}\left(1-\frac{1-\alpha}{\sqrt{(1-\alpha)^{2}+\tau^{2}}}+O_{\varepsilon}\left(\frac{1}{u}+e^{-(\log y)^{\varepsilon / 2}}\right)\right)-c_{4} y^{1 / 2-\alpha} \\
& \gg u\left(\frac{\tau^{2}}{(1-\alpha)^{2}+\tau^{2}}+O_{\varepsilon}\left(\frac{1}{u}+\exp \left\{-(\log y)^{\varepsilon / 2}\right\}\right)\right) .
\end{aligned}
$$

From (3.18) we easily see that in the ranges considered

$$
\frac{\tau^{2}}{(1-\alpha)^{2}+\tau^{2}} \gg \min \left(1,\left(\frac{\tau}{1-\alpha}\right)^{2}\right) \gg(\log u)^{-2} \geq(\log y)^{-2}
$$

Thus if $y_{0}(\varepsilon)$ and $u_{0}(\varepsilon)$ are suitably chosen, we deduce that

$$
W \gg u \tau^{2} /\left((1-\alpha)^{2}+\tau^{2}\right) \quad(y>\log x) .
$$

This completes the proof of (3.16). 
LEMMA 9. We have uniformly for $x \geq y \geq 2,0<\varepsilon<1,1 \leq z \leq Y(\varepsilon)$,

$$
\Psi(x+x / z, y)-\Psi(x, y) \ll_{\varepsilon} x^{\alpha} \zeta(\alpha, y)\left\{1 / z+e^{-c_{5} \bar{u}}\right\}
$$

PrOOF. The left-hand side of (3.19) is

$$
O\left(\sum_{\substack{n \geq 1 \\ P(n) \leq y}} \exp \left\{-\frac{1}{2}\left(z \log \left(\frac{x}{n}\right)\right)^{2}\right\}\right) .
$$

We express the exponential in terms of its bilateral Laplace transform using the formula

$$
\begin{aligned}
e^{-v^{2} / 2} & =\frac{1}{i \sqrt{2 \pi}} \int_{\sigma-i \infty}^{\sigma+i \infty} \exp \left\{\frac{s^{2}}{2}-v s\right\} d s \\
& =\frac{1}{\sqrt{2 \pi}} e^{\sigma^{2} / 2-\sigma v} \int_{-\infty}^{+\infty} \exp \left\{-\frac{1}{2} \tau^{2}+i \tau(\sigma-v)\right\} d \tau \quad(\sigma, v \in \mathbf{R}) .
\end{aligned}
$$

Selecting $\sigma=\alpha(x, y) / z$, we get

$$
\begin{aligned}
\frac{1}{\sqrt{2 \pi}} e^{\alpha^{2} / 2 z^{2}} \sum_{\substack{n \geq 1 \\
P(n) \leq y}} \int_{-\infty}^{+\infty} e^{i \alpha \tau / z-\tau^{2} / 2}\left(\frac{x}{n}\right)^{\alpha+i \tau z} d \tau \\
=\frac{1}{\sqrt{2 \pi}} e^{\alpha^{2} / 2 z^{2}} \int_{-\infty}^{+\infty} e^{i \alpha \tau / z-\tau^{2} / 2} \sum_{\substack{n \geq 1 \\
P(n) \leq y}}\left(\frac{x}{n}\right)^{\alpha+i \tau z} d \tau \\
\ll \frac{x^{\alpha}}{z} \int_{-\infty}^{+\infty} e^{-\tau^{2} / 2 z^{2}}|\zeta(\alpha+i \tau, y)| d \tau,
\end{aligned}
$$

the inversion of summations being justified by the absolute convergence of the Dirichlet series involved. In the last integral, we divide the range of integration into three parts: $|\tau| \leq 1,1 \leq|\tau| \leq z^{2}$, and $|\tau|>z^{2}$. The corresponding contributions $I_{1}, I_{2}, I_{3}$ can be estimated as follows.

$$
\begin{aligned}
I_{1} & \ll \zeta(\alpha, y) \int_{-1}^{1} e^{-\tau^{2} / 2 z^{2}} d \tau \ll \zeta(\alpha, y), \\
I_{2} & \ll \sup _{1 \leq|\tau| \leq z^{2}}|\zeta(\alpha+i \tau, y)| \int_{1}^{z^{2}} e^{-\tau^{2} / 2 z^{2}} d \tau \\
& \ll z \sup _{1 \leq|\tau| \leq z^{2}}|\zeta(\alpha+i \tau, y)| \ll_{\varepsilon} z \zeta(\alpha, y) e^{-c_{5} \bar{u}}
\end{aligned}
$$

by Lemma 8 , and

$$
I_{3} \ll \varsigma(\alpha, y) \int_{z^{2}}^{\infty} e^{-\tau^{2} / 2 z^{2}} d \tau \ll \varsigma(\alpha, y) .
$$

This provides the estimate wanted. 
4. Proof of Theorem 1. The analytic method sketched in $\S 2$ works well if $u$ is large, but complications arise when $u$ is smaller than some fixed power of $\log \log y$. We shall therefore use this method only for the range

$$
u \geq(\log \log 2 y)^{2} .
$$

In the range $1 \leq u \leq(\log \log 2 y)^{2}$, de Bruijn's estimate (1.5) is available and implies estimate (2.3) of Theorem 1 via formulae (2.6) and (2.7). Note that formula (2.7), which we stated as a corollary to Theorems 1 and 2, actually depends only on Theorem 2 and the special case $u=\sqrt{\log 2 y}$ of Theorem 1, which is covered by (4.1). Theorem 2 (and hence (2.6)) will be established in the last section without using Theorem 1.

It remains therefore to prove Theorem 1 in the case when (4.1) is satisfied. This is the really interesting and difficult case. We give the main steps of the proof in the form of two lemmas.

LEMMA 10. Let $\varepsilon, 0<\varepsilon<1$, be fixed. We have uniformly for $x \geq y \geq 2$,

$$
\begin{aligned}
\Psi(x, y)= & \frac{1}{2 i \pi} \int_{\alpha-i / \log y}^{\alpha+i / \log y} \varsigma(s, y) \frac{x^{s}}{s} d s \\
& +O_{\varepsilon}\left(x^{\alpha} \varsigma(\alpha, y)\left(Y(\varepsilon)^{-1}+\exp \left\{-c_{6} u(\log 2 u)^{-2}\right\}\right)\right) .
\end{aligned}
$$

LEMMA 11. We have uniformly for $x \geq y \geq 2$,

$$
\frac{1}{2 i \pi} \int_{\alpha-i / \log y}^{\alpha-i / \log y} \varsigma(s, y) \frac{x^{s}}{s} d s=\frac{x^{\alpha} \varsigma(\alpha, y)}{\alpha \sqrt{2 \pi \phi_{2}(\alpha, y)}}\left(1+O\left(\frac{1}{\bar{u}}\right)\right) \text {. }
$$

Moreover, the same estimate holds for

$$
\frac{1}{2 \pi} \int_{\alpha-i / \log y}^{\alpha+i / \log y}\left|\varsigma(s, y) \frac{x^{s}}{s}\right| \cdot|d s| .
$$

The desired estimate (2.3) of Theorem 1 follows easily from these lemmas when we assume (4.1). We take $\varepsilon=1 / 4$ and combine formulae (4.2) and (4.3). The right-hand side of (4.3) coincides with the estimate announced for $\Psi(x, y)$ (since $1 / u+(\log y) / y \asymp 1 / \bar{u})$, so it only remains to check that the error in (4.2) is absorbed by the error terms in (2.3). This is an immediate consequence of the bound

$$
\alpha \sqrt{\phi_{2}(\alpha, y)} \ll \min (\sqrt{u} \log y, \sqrt{y / \log y})
$$

(which follows from Lemmas 2, 4) under our hypothesis (4.1).

We now prove the two lemmas. In view of (4.4), the conclusions are trivial when $y \leq y_{0}$, so we assume $y>y_{0}$ henceforth.

PROOF OF LEMMA 10 . We start with the formula

$$
\begin{aligned}
\Psi(x, y)= & \frac{1}{2 i \pi} \int_{\sigma-i T}^{\sigma+i T} \varsigma(s, y) \frac{x^{s}}{s} d s \\
& +O\left(x^{\sigma} \sum_{\substack{n \geq 1 \\
P(n) \leq y}} \frac{1}{n^{\sigma}} \min \left(1, \frac{1}{T|\log (x / n)|}\right)\right),
\end{aligned}
$$


valid uniformly for $\sigma>0$ and $T>0$ (we set here $\min (1,1 / 0)=1$ ). This is a variant of Perron's formula and follows from the estimate

$$
\frac{1}{2 i \pi} \int_{\sigma-i T}^{\sigma+i T} \frac{z^{s}}{s} d s=\chi(z)+O\left(z^{\sigma} \min \left(1, \frac{1}{T|\log z|}\right)\right)
$$

where

$$
\chi(z)= \begin{cases}0 & (0<z \leq 1) \\ 1 & (z>1)\end{cases}
$$

which is easily proved by contour integration.

We select $\sigma=\alpha(x, y), T=\left(Y(\varepsilon)^{-1}+\exp \left(-c_{6} u(\log 2 u)^{-2}\right)\right)^{-2}$. The error term in (4.5) may be estimated by

$$
O\left(\frac{x^{\alpha} \varsigma(\alpha, y)}{\sqrt{T}}+x^{\alpha} \sum_{\substack{P(n) \leq y \\|\log (x / n)| \leq T^{-1 / 2}}} n^{-\alpha}\right)
$$

The first term is compatible with the size of the error in (4.2). The second is

$$
O\left(\Psi\left(x+\frac{c_{7} x}{\sqrt{T}}, y\right)-\Psi\left(x-\frac{c_{7} x}{\sqrt{T}}, y\right)\right) \ll_{\varepsilon} x^{\alpha} \zeta(\alpha, y)\left\{\frac{1}{\sqrt{T}}+e^{-c_{5} \bar{u}}\right\}
$$

by Lemma 9 . This is also acceptable.

It remains to estimate the contribution of the domain $1 / \log y<|\tau| \leq T$. By Lemma 8 , this is

$$
\begin{gathered}
\ll_{\varepsilon} x^{\alpha} \zeta(\alpha, y) \int_{1 / \log y}^{T} e^{-c_{0} \bar{u} \tau^{2} /\left((1-\alpha)^{2}+\tau^{2}\right)} \frac{d \tau}{\alpha+\tau} \\
\ll_{\varepsilon} x^{\alpha} \zeta(\alpha, y)\left\{\int_{1 / \log y}^{\max (|1-\alpha|, 1 / \log y)} e^{-c_{0} \bar{u} \tau^{2} / 2(1-\alpha)^{2}} \frac{d \tau}{\alpha+\tau}\right. \\
\left.+\int_{\max (|1-\alpha|, 1 / \log y)}^{T} e^{-c_{0} \bar{u} / 2} \frac{d \tau}{\alpha+\tau}\right\} \\
\ll_{\varepsilon} x^{\alpha}{ }_{\zeta}(\alpha, y)\left\{e^{-c_{0} \bar{u} / 2((1-\alpha) \log y)^{2}} \log \left(1+\frac{1}{\alpha+1 / \log y}\right)+e^{-c_{0} \bar{u} / 2} \log T\right\} .
\end{gathered}
$$

Now, we notice that Lemma 3 implies that

$$
\log \left(1+\frac{1}{\alpha+1 / \log y}\right), \quad(1-\alpha) \log y \ll \log 2 \bar{u} .
$$

Substituting in the last estimate, we see that the remainder term above is

$$
\begin{aligned}
& \ll_{\varepsilon} x^{\alpha} \zeta(\alpha, y) \exp \left(-c_{7} \bar{u}(\log 2 \bar{u})^{-2}\right) \log T \\
& \ll_{\varepsilon} x^{\alpha} \zeta(\alpha, y) T^{-1 / 2},
\end{aligned}
$$

if $c_{6}$ has been chosen sufficiently small.

This completes the proof.

PROOF OF LEMMA 11. We divide the range of integration in (4.3) into the parts $|\tau| \leq T_{0}$ and $T_{0}<|\tau| \leq 1 / \log y$, where $T_{0}=(\bar{u})^{2 / 3} /(u \log y)$. 
The main contribution comes from the range $|\tau| \leq T_{0}$. Here we expand the function $\phi(s, y)=\log \varsigma(s, y)$ in a Taylor series around $\tau=0$. Putting $\sigma_{k}=\phi_{k}(\alpha, y)(k \geq$ $0)$, we get

$$
\phi(s, y)=\sigma_{0}+i \tau \sigma_{1}-\frac{\tau^{2}}{2} \sigma_{2}-i \frac{\tau^{3}}{3 !} \sigma_{3}+O\left(\tau^{4} \sigma_{4}\right)
$$

where the error term estimate follows from the inequality

$$
\sup _{\tau \in \mathbf{R}}\left|\phi_{4}(\alpha+i \tau, y)\right| \leq \phi_{4}(\alpha, y)=\sigma_{4}
$$

which is an obvious consequence of the "explicit" formula (3.9) for $\phi_{k}(s, y)$.

By the definition of $T_{0}$ and Lemmas 2, 4, the quantities $\tau^{3} \sigma_{3}, \tau^{4} \sigma_{4}$ and $\tau / \alpha$ are uniformly bounded for $|\tau| \leq T_{0}$. Thus, we have in this range

$$
\exp \left\{-i \frac{\tau^{3}}{3 !} \sigma_{3}+O\left(\tau^{4} \sigma_{4}\right)\right\}=1-i \frac{\tau^{3}}{3 !} \sigma_{3}+O\left(\tau^{6} \sigma_{3}^{2}+\tau^{4} \sigma_{4}\right)
$$

and

$$
\frac{1}{\alpha+i \tau}=\frac{1}{\alpha}\left(1-i \frac{\tau}{\alpha}+O\left(\frac{\tau^{2}}{\alpha^{2}}\right)\right) \text {. }
$$

Bearing in mind that, by definition of $\alpha, \sigma_{1}+\log x=0$, we obtain the following expression for the integrand in (4.3):

$$
\begin{aligned}
\varsigma(s, y) \frac{x^{s}}{s} & =\frac{\varsigma(\alpha, y) x^{\alpha}}{\alpha+i \tau} \exp \left\{i \tau \log x+i \tau \sigma_{1}-\frac{\tau^{2}}{2} \sigma_{2}-i \frac{\tau^{3}}{3 !} \sigma_{3}+O\left(\tau^{4} \sigma_{4}\right)\right\} \\
& =\frac{x^{\alpha} \varsigma(\alpha, y)}{\alpha} e^{-\tau^{2} \sigma_{2} / 2}\left\{1-i \frac{\tau}{\alpha}-i \frac{\tau^{3}}{3 !} \sigma_{3}+O\left(\tau^{6} \sigma_{3}^{2}+\tau^{2} \alpha^{-2}+\tau^{4} \sigma_{4}\right)\right\}
\end{aligned}
$$

We now integrate the last expression over the range $|\tau| \leq T_{0}$. Omitting the constant factor $x^{\alpha} \zeta(\alpha, y) / \alpha$ gives for the contribution of the main terms

$$
\int_{-T_{0}}^{T_{0}} e^{-\tau^{2} \sigma_{2} / 2} d \tau=\frac{1}{\sqrt{\sigma_{2}}} \int_{-T_{0} \sqrt{\sigma_{2}}}^{T_{0} \sqrt{\sigma_{2}}} e^{-\tau^{2} / 2} d \tau=\sqrt{\frac{2 \pi}{\sigma_{2}}}\left(1+O\left(\exp \left\{-\frac{1}{2} T_{0}^{2} \sigma_{2}\right\}\right)\right)
$$

since the integrals involving $i \tau / \alpha$ and $i \tau^{3} \sigma_{3} / 3$ ! vanish. The error terms contribute at most

$$
O\left(\frac{1}{\sqrt{\sigma_{2}}}\left(\sigma_{2}^{-3} \sigma_{3}^{2}+\sigma_{2}^{-1} \alpha^{-2}+\sigma_{2}^{-2} \sigma_{4}\right)\right)
$$

By Lemmas 2, 4, we have

$$
\alpha \gg \bar{u} /(u \log y), \quad \sigma_{k} \asymp(u \log y)^{k}(\bar{u})^{1-k} \quad(k=2,3,4) .
$$

The factor $1 / \sqrt{\sigma_{2}}$ in the above expression is therefore $O(1 / \bar{u})$. Moreover, from the definition of $T_{0}$ we also obtain

$$
\exp \left\{-\frac{1}{2} T_{0}^{2} \sigma_{2}\right\} \ll\left(T_{0}^{2} \sigma_{2}\right)^{-3} \asymp(\bar{u})^{-1}
$$

Collecting the above estimates yields

$$
\frac{1}{2 i \pi} \int_{-T_{0}}^{T_{0}} \varsigma(s, y) \frac{x^{s}}{s} d s=\frac{x^{\alpha} \varsigma(\alpha, y)}{\alpha \sqrt{2 \pi \sigma_{2}}}\left(1+O\left(\frac{1}{\bar{u}}\right)\right)
$$

which is the right-hand side of (4.3). 
It only remains to show that the contribution of the range $T_{0}<|\tau| \leq 1 / \log y$ can be absorbed by the error term. By Lemma 8(i), we have in this range

$$
\varsigma(s, y) \frac{x^{s}}{s} \ll \varsigma(\alpha, y) \frac{x^{\alpha}}{\alpha}\left(1+\frac{\tau^{2} \sigma_{2} \log y}{y}\right)^{-c_{0} y / \log y} .
$$

Thus the corresponding contribution may be estimated as follows (omitting again the constant factor $\left.\zeta(\alpha, y) x^{\alpha} / \alpha\right)$ :

$$
\begin{aligned}
& \ll \frac{1}{\sqrt{\sigma_{2}}} \int_{T_{0} \sqrt{\sigma_{2}}}^{\infty}\left(1+\frac{\tau^{2} \log y}{y}\right)^{-c_{0} y / \log y} d \tau \\
& \ll \frac{1}{\sqrt{\sigma_{2}}}\left\{\int_{T_{0} \sqrt{\sigma_{2}}}^{\sqrt{y / 2 \log y}} e^{-c_{0} \tau^{2} / 2} d \tau\right. \\
& \left.\quad+\int_{\sqrt{y / 2 \log y}}^{\infty}\left(1+\frac{\tau^{2} \log y}{y}\right)^{-c_{0} y / \log y} d \tau\right\} \\
& \ll \frac{1}{\sqrt{\sigma_{2}}}\left(T_{0}^{-6} \sigma_{2}^{-3}+\log y \cdot y^{-1}\right) \ll \frac{1}{\sqrt{\sigma_{2}} \cdot \bar{u}} .
\end{aligned}
$$

This completes the proof of (4.3).

An inspection of the proof shows that we obtain the same estimate when the integral is taken over the absolute value, as asserted in the lemma. We omit the details.

5. Proof of Theorem 4. This is a simple modification of the argument leading to Theorem 1. We again use Lemma 10 together with the following result, which can be established in the same way as Lemma 11.

LEMMA 12. We have uniformly for $x \geq y \geq 2$,

$$
\frac{1}{2 i \pi} \int_{\alpha-i / \log y}^{\alpha+i / \log y} \varsigma(s, y) x^{s} d s=\frac{x^{\alpha} \zeta(\alpha, y)}{\sqrt{2 \pi \phi_{2}(\alpha, y)}}\left(1+O\left(\frac{1}{\bar{u}}\right)\right) \text {. }
$$

Moreover the same estimate holds for

$$
\frac{1}{2 \pi} \int_{\alpha-i / \log y}^{\alpha+i / \log y}\left|\zeta(s, y) x^{s}\right| \cdot|d s| .
$$

Put $x^{\prime}=x(1+1 / z), \alpha^{\prime}=\alpha\left(x^{\prime}, y\right)$. Differentiating the equation $\phi_{1}(\alpha, y)=-\log x$ with respect to $x$ yields

whence, by Lemma 4,

$$
\frac{\partial \alpha}{\partial x}(x, y)=\frac{-1}{x \phi_{2}(\alpha, y)}
$$

$$
O<-\frac{\partial \alpha}{\partial x}(x, y) \asymp \frac{\bar{u}}{x u^{2}(\log y)^{2}}
$$

and

$$
O \leq \alpha-\alpha^{\prime} \ll \frac{\bar{u}}{z u^{2}(\log y)^{2}} \leq \frac{1}{u(\log y)^{2}}
$$

In particular, we have for all real $\tau$ and $\beta, \alpha^{\prime} \leq \beta \leq \alpha$,

$$
\left(x^{\prime}\right)^{\beta}|\zeta(\beta+i \tau, y)| \asymp x^{\alpha}|\zeta(\alpha+i \tau, y)| \text {. }
$$


Using this for $\tau=0$ and applying Lemma 10 to estimate $\Psi\left(x^{\prime}, y\right)$, we obtain

$$
\Psi\left(x^{\prime}, y\right)=\frac{1}{2 i \pi} \int_{\alpha^{\prime}-i / \log y}^{\alpha^{\prime}+i / \log y} \varsigma(s, y) \frac{x^{\prime s}}{s} d s+O_{\varepsilon}\left(x^{\alpha} \zeta(\alpha, y) R^{\prime}(x, y)\right)
$$

with

$$
R^{\prime}(x, y)=Y(\varepsilon)^{-1}+\exp \left\{-c_{6} u(\log 2 u)^{-2}\right\} .
$$

Now (5.1) and the residue-theorem imply that the error involved in replacing $\alpha^{\prime}$ by $\alpha$ in the main term of $(5.2)$ is

$$
\ll \frac{|\zeta(\alpha+i / \log y, y)| x^{\alpha}}{\alpha+1 / \log y}\left|\alpha^{\prime}-\alpha\right| \ll \zeta(\alpha, y) x^{\alpha} R^{\prime}(x, y),
$$

where the second estimate follows from Lemma 8(i) with a suitable modification of the constant $c_{6}$. Thus, we have

$$
\Psi\left(x^{\prime}, y\right)=\frac{1}{2 i \pi} \int_{\alpha-i / \log y}^{\alpha+i / \log y} \varsigma(x, y) \frac{x^{\prime s}}{s} d s+O_{\varepsilon}\left(x^{\alpha} \varsigma(\alpha, y) R^{\prime}(x, y)\right) .
$$

Subtracting (4.2), we obtain

$$
\begin{aligned}
\Psi\left(x+\frac{x}{z}, y\right)-\Psi(x, y)= & \frac{1}{2 i \pi} \int_{\alpha-i / \log y}^{\alpha+i / \log y} \varsigma(x, y) x^{s}\left(\frac{(1+1 / z)^{s}-1}{s}\right) d s \\
& +O_{\varepsilon}\left(x^{\alpha} \zeta(\alpha, y) R^{\prime}(x, y)\right) .
\end{aligned}
$$

By Theorem 1 and equation (4.4), we easily obtain that the remainder term is $O_{\varepsilon}(\Psi(x, y) R(x, y))$.

Using the expansion

$$
(1+1 / z)^{s}=1+s / z+O\left(|s| / z^{2}\right) \quad(z \geq 1,|s| \ll 1)
$$

we find that the main term equals

$$
\frac{1}{2 i \pi z} \int_{\alpha-i / \log y}^{\alpha+i / \log y} \zeta(s, y) x^{s} d s+O\left(\frac{1}{z^{2}} \int_{\alpha-i / \log y}^{\alpha+i / \log y}\left|\zeta(s, y) x^{s}\right| \cdot|d s|\right) .
$$

By Lemma 12, this is

$$
\frac{x^{\alpha} \varsigma(\alpha, y)}{z \sqrt{2 \pi \phi_{2}(\alpha, y)}}\left(1+O\left(\frac{1}{\bar{u}}+\frac{1}{z}\right)\right)
$$

and, by Theorem 1, this last expression may also be written as

$$
\frac{\alpha \Psi(x, y)}{z}\left(1+O\left(\frac{1}{\bar{u}}+\frac{1}{z}\right)\right)
$$

This completes the proof of Theorem 4 .

6. Proof of Theorem 3. Theorem 3 could be proved directly in the same manner as Theorem 4, but it is easier to deduce it from estimate (2.3) of Theorem 1.

We first dispose of the cases $2 \leq y \leq y_{0}$ and $1 \leq u \leq u_{0}$, where $y_{0}$ and $u_{0}$ are arbitrary but fixed constants. All we have to show then is the upper bound

$$
\Psi(c x, y) / \Psi(x, y) \ll c^{\alpha(x, y)} \quad(1 \leq c \leq y) .
$$


When $2 \leq y \leq y_{0}$, this follows from (1.9). In the case $y>y_{0}, 1 \leq u \leq u_{0}$, with $y_{0}$ sufficiently large, (6.1) can be deduced from (1.5) and the estimate $\alpha(x, y)=$ $1+O(1 / \log y)\left(y \leq x \leq y^{u_{0}}\right)$, which follows easily from the equation defining $\alpha$.

We may therefore suppose

$$
y \geq y_{0}, \quad u \geq u_{0}
$$

with sufficiently large constants $y_{0}, u_{0}$.

Let $y \geq y_{0}$ be fixed and put for $u \geq 1$,

$$
\alpha_{u}=\alpha\left(y^{u}, y\right)
$$

and

$$
\begin{aligned}
f(u) & =\log \left(\frac{y^{u \alpha_{u}} \zeta\left(\alpha_{u}, y\right)}{\alpha_{u} \sqrt{2 \pi \phi_{2}\left(\alpha_{u}, y\right)}}\right) \\
& =u \alpha_{u} \log y+\phi\left(\alpha_{u}, y\right)-\log \alpha_{u}-\frac{1}{2} \log \left(2 \pi \phi_{2}\left(\alpha_{u}, y\right)\right) .
\end{aligned}
$$

Thus, exp $f(u)$ is the approximation of Theorem 1 for $\Psi\left(y^{u}, y\right)$ and, under hypothesis (6.2), estimate (2.3) of Theorem 1 can be restated as

$$
\Psi\left(y^{u}, y\right)=\exp \{f(u)+O(1 / \bar{u})\}
$$

Estimate (2.9) of Theorem 3 is therefore, in the range (6.2), equivalent to the formula

$$
f(u+t)-f(u)=t \alpha_{u} \log y+O(1 / \bar{u}) \quad\left(u \geq u_{0}, 0 \leq t \leq 1\right) .
$$

Now,

$$
f(u+t)=f(u)+t f^{\prime}(u)+O\left(\sup _{0 \leq t \leq 1}\left|f^{\prime \prime}(u+t)\right|\right)
$$

uniformly for $u \geq 1,0 \leq t \leq 1$. Hence (6.3) is a consequence of the two estimates

$$
f^{\prime}(u)=\alpha_{u} \log y+O(1 / \bar{u}) \quad\left(u \geq u_{0}\right)
$$

and

$$
f^{\prime \prime}(u)=O(1 / \bar{u}) \quad\left(u \geq u_{0}\right)
$$

which we are going to prove.

We first derive estimates for the derivatives $\alpha_{u}^{\prime}, \alpha_{u}^{\prime \prime}$ of $\alpha_{u}=\alpha\left(y^{u}, y\right)$, considered as a function of $u$. Differentiating the equation

$$
u \log y+\phi_{1}\left(\alpha_{u}, y\right)=0
$$

yields

$$
\alpha_{u}^{\prime}=-(\log y) / \phi_{2}\left(\alpha_{u}, y\right) \quad \text { and } \quad \alpha_{u}^{\prime \prime}=-\phi_{3}\left(\alpha_{u}, y\right)\left(\alpha_{u}^{\prime}\right)^{2} / \phi_{2}\left(\alpha_{u}, y\right) \text {. }
$$

An application of Lemmas 2, 4 then gives

$$
\begin{aligned}
& \left|\alpha_{u}^{\prime}\right| \asymp \bar{u} /\left(u^{2} \log y\right), \\
& \left|\alpha_{u}^{\prime \prime}\right| \asymp \bar{u} /\left(u^{3} \log y\right),
\end{aligned}
$$


Differentiating the expression defining $f(u)$, we find

$$
\begin{aligned}
f^{\prime}(u) & =\alpha_{u} \log y+u \alpha_{u}^{\prime} \log y+\phi_{1}\left(\alpha_{u}, y\right) \alpha_{u}^{\prime}-\frac{\alpha_{u}^{\prime}}{\alpha_{u}}-\frac{\phi_{3}\left(\alpha_{u}, y\right) \alpha_{u}^{\prime}}{2 \phi_{2}\left(\alpha_{u}, y\right)} \\
& =\alpha_{u} \log y-\frac{\alpha_{u}^{\prime}}{\alpha_{u}}-\frac{\phi_{3}\left(\alpha_{u}, y\right) \alpha_{u}^{\prime}}{2 \phi_{2}\left(\alpha_{u}, y\right)}
\end{aligned}
$$

and

$$
\begin{aligned}
f^{\prime \prime}(u)= & \alpha_{u}^{\prime} \log y-\frac{\alpha_{u} \alpha_{u}^{\prime \prime}-\left(\alpha_{u}^{\prime}\right)^{2}}{\alpha_{u}^{2}} \\
& -\frac{\phi_{2}\left(\alpha_{u}, y\right)\left(\phi_{4}\left(\alpha_{u}, y\right) \alpha_{u}^{\prime 2}+\phi_{3}\left(\alpha_{u}, y\right) \alpha_{u}^{\prime \prime}\right)-\phi_{3}\left(\alpha_{u}, y\right) \phi_{2}\left(\alpha_{u}, y\right)}{2 \phi_{2}\left(\alpha_{u}, y\right)} .
\end{aligned}
$$

Inserting (6.6), (6.7), and the estimates

$$
\alpha_{u} \gg \bar{u} /(u \log y), \quad\left|\phi_{k}\left(\alpha_{u}, y\right)\right| \asymp(u \log y)^{k}(\bar{u})^{1-k} \quad(k=2,3,4),
$$

of Lemmas 2, 4, we obtain (6.4) and (6.5), and hence the assertion of Theorem 3 in the form (6.3).

7. Proof of Theorem 2. The proof is somewhat lengthy but not difficult. We start with a lemma giving relatively sharp estimates for $\phi_{1}(\sigma, y)$ and $\phi_{2}(\sigma, y)$.

LEMMA 13. We have uniformly for $y \geq 2$ and $\sigma>0$,

$$
-\phi_{1}(\sigma, y)=\left(1+O\left(\frac{1}{\log y}\right)\right) \frac{1}{1-y^{-\sigma}} \int_{1}^{y} \frac{d t}{t^{\sigma}}+O(1)
$$

and

$$
\phi_{2}(\sigma, y)=\left(1+O\left(\frac{1}{\log y}\right)\right) \frac{1}{\left(1-y^{-\sigma}\right)^{2}} \int_{1}^{y} \frac{\log t}{t^{\sigma}} d t+O(1) .
$$

Moreover, for any fixed positive constants $\varepsilon$ and $\sigma_{0}$, the error terms $O(1 / \log y)$ can be replaced by $O_{\varepsilon, \sigma_{0}}\left(\exp \left\{-(\log y)^{3 / 5-\varepsilon}\right\}\right)$ in the case $\sigma \geq \sigma_{0}$.

ProOF. The case $2 \leq y<3$ being trivial, let $y \geq 3$ be fixed and put

$$
R:=1 / \log y, \quad R_{\varepsilon}:=\exp \left\{-(\log y)^{3 / 5-\varepsilon}\right\} \quad(0<\varepsilon<1 / 2) .
$$

By partial summation and a strong form of the prime number theorem, we readily get

$$
-\phi_{1}(\sigma, y)=\sum_{p \leq y} \frac{\log p}{p^{\sigma}-1}=\left(1+O_{\varepsilon}\left(R_{\varepsilon}\right)\right) \int_{2}^{y} \frac{d t}{t^{\sigma}-1}+O(1)
$$

uniformly for $\sigma>0$ and any fixed $\varepsilon>0$. Thus to obtain the asserted estimates concerning $\phi_{1}(\sigma, y)$, it suffices to show

$$
\int_{2}^{y} \frac{d t}{t^{\sigma}-1}=\frac{1+O(R)}{1-y^{-\sigma}} \int_{1}^{y} \frac{d t}{t^{\sigma}}+O(1)
$$

and, when $\sigma \geq \sigma_{0}$,

$$
\int_{2}^{y} \frac{d t}{t^{\sigma}-1}=\frac{1+O_{\varepsilon, \sigma_{0}}\left(R_{\varepsilon}\right)}{1-y^{-\sigma}} \int_{1}^{y} \frac{d t}{t^{\sigma}}+O(1)
$$


Since

we obtain

$$
\frac{1}{t^{\sigma}-1}=\frac{1}{t^{\sigma}}+\frac{1}{t^{2 \sigma}\left(1-t^{-\sigma}\right)}
$$

$$
\int_{2}^{y} \frac{d t}{t^{\sigma}-1}=\int_{1}^{y} \frac{d t}{t^{\sigma}}+O\left(\frac{1}{1-2^{-\sigma}} \int_{1}^{y} \frac{d t}{t^{2 \sigma}}\right) .
$$

The error term is bounded if $\sigma \geq 2 / 3$ and is smaller than the main term by a factor

$$
O\left(((\log y) / \sigma) y^{-\min (\sigma, 1 / 3)}\right)
$$

if $0<\sigma \leq 2 / 3$. This is $O_{\varepsilon}\left(R_{\varepsilon}\right)$ if $\sigma \geq \sigma_{0}$ and $O(R)$ if $\sigma \geq 3(\log \log 2 y) / \log y$. Thus, it only remains to prove (7.3) in the case $\sigma \leq \min (2 / 3,3(\log \log 2 y) / \log y)$.

In this range we have

$$
\begin{aligned}
\int_{1}^{y} \frac{d t}{t^{\sigma}} & =\frac{y^{1-\sigma}-1}{1-\sigma}=\frac{y^{1-\sigma}}{1-\sigma}\left(1+O\left(y^{-1 / 3}\right)\right) \\
& =\left(1+O\left(y^{-1 / 6}\right)\right) \int_{\sqrt{y}}^{y} \frac{d t}{t^{\sigma}}
\end{aligned}
$$

and

$$
\begin{aligned}
\int_{2}^{y} \frac{d t}{t^{\sigma}-1} & =\int_{\sqrt{y}}^{y} \frac{d t}{t^{\sigma}-1}+O\left(\frac{1}{1-2^{-\sigma}} \int_{1}^{\sqrt{y}} \frac{d t}{t^{\sigma}}\right) \\
& =\left(1+O\left(y^{-1 / 6} \log y\right)\right) \int_{\sqrt{y}}^{y} \frac{d t}{t^{\sigma}-1}
\end{aligned}
$$

To obtain (7.3), it therefore suffices to show

$$
\int_{\sqrt{y}}^{y} \frac{1}{t^{\sigma}}\left(\frac{1}{1-t^{-\sigma}}-\frac{1}{1-y^{-\sigma}}\right) d t \ll \frac{y^{1-\sigma}}{1-y^{-\sigma}} R .
$$

The left-hand side equals

$$
\begin{aligned}
& \frac{1}{1-y^{-\sigma}} \int_{\sqrt{y}}^{y} \frac{1-(y / t)^{-\sigma}}{t^{2 \sigma}\left(1-t^{-\sigma}\right)} d t \\
& \quad \leq \frac{\sigma}{\left(1-y^{-\sigma}\right)\left(1-y^{-\sigma / 2}\right)} \int_{\sqrt{y}}^{y} \frac{\log (y / t)}{t^{2 \sigma}} d t \\
& \quad \ll \frac{\sigma y^{1-2 \sigma}}{\left(1-y^{-\sigma}\right) \min (1, \sigma \log y)} \ll \frac{y^{1-\sigma}}{1-y^{-\sigma}} R .
\end{aligned}
$$

This establishes the estimates of the lemma for $\phi_{1}(\alpha, y)$. The results concerning $\phi_{2}(\alpha, y)$ are proved similarly.

PROOF OF THEOREM 2. When $y$ is bounded, the result follows by trivial arguments. Hence, we may suppose $y \geq y_{0}$, where $y_{0}$ is a sufficiently large absolute constant.

Let $x_{0}=e^{y_{0}}$ and $\varepsilon$ be any positive constant. We are going to prove

$$
\alpha(x, y)=\frac{\log (1+y / \log x)}{\log y}\left(1+O\left(\frac{1}{\log y}\right)\right)
$$

for

$$
y_{0} \leq y \leq(\log x)^{2}
$$


and

$$
\alpha(x, y)=1-\frac{\xi(u)}{\log y}+O_{\varepsilon}\left(R_{\varepsilon}+\frac{1}{u(\log y)^{2}}\right)
$$

for

$$
x \geq x_{0}, \quad(\log x)^{1+\varepsilon}<y \leq x .
$$

Here, as in the previous section, we set

$$
R_{\varepsilon}=\exp \left\{-(\log y)^{3 / 5-\varepsilon}\right\} .
$$

We first check that (7.6) and (7.8) together imply estimate (2.4) of Theorem 2. Since the right-hand side of (7.6) is identical with the right-hand side of $(2.4)$ except for the sharper remainder, we only have to consider the range (7.9). By Lemma 1 , we have in this region

$$
\begin{aligned}
\xi(u) & =\log u+O(\log \log (u+2)) \\
& =\log \log x+O(\log \log (y+1)),
\end{aligned}
$$

whence from (7.8)

$$
\begin{aligned}
\alpha(x, y) & =\frac{\log (y / \log x)}{\log y}+O\left(\frac{\log \log (y+1)}{\log y}\right) \\
& =\frac{\log (1+y / \log x)}{\log y}\left(1+O_{\varepsilon}\left(\frac{\log \log (y+1)}{\log y}\right)\right),
\end{aligned}
$$

as wanted.

To prove (7.6) and (7.8), we introduce as before, for fixed $y \geq y_{0}$, the functions

$$
\alpha_{u}=\alpha\left(y^{u}, y\right), \quad \alpha_{u}^{\prime}=d \alpha_{u} / d u
$$

and, given $u=(\log x) /(\log y)$, define $v$ and $w$ by the equations

$$
\alpha_{v}=\frac{\log (1+y /(\log x))}{\log y}, \quad \alpha_{w}=1-\frac{\xi(u)}{\log y} .
$$

Thus we have to prove that

$$
\left|\alpha_{u}-\alpha_{v}\right| \ll \alpha_{v} / \log y
$$

and

$$
\left|\alpha_{u}-\alpha_{w}\right| \ll R_{\varepsilon}+1 / u(\log y)^{2}
$$

hold respectively in the ranges (7.7) and (7.9).

By Lemma 13 we have for any positive $z$,

$$
-\phi_{1}\left(y, \alpha_{z}\right)=z \log y=\frac{y\left(1-y^{\alpha_{z}-1}\right)}{\left(y^{\alpha_{z}}-1\right)\left(1-\alpha_{z}\right)}\left(1+O\left(\frac{1}{\log y}\right)\right)+O(1)
$$

where the error term $O(1 / \log y)$ may be replaced by $O_{\varepsilon}\left(R_{\varepsilon}\right)$ if $\alpha_{z} \gg_{\varepsilon} 1$. From the definitions of $\alpha_{v}$ and $\alpha_{w}$, we have

$$
\frac{y}{y^{\alpha_{v}}-1}=u \log y, \quad \frac{y^{1-\alpha_{w}}-1}{1-\alpha_{w}}=u \log y .
$$


Moreover, we have in the range (7.7),

$$
0<\alpha_{v} \leq \frac{\log \left(1+y^{1 / 2}\right)}{\log y}<\frac{3}{4}, \quad \text { say }
$$

and in the range (7.9),

$$
1 \ll_{\varepsilon} \quad \alpha_{w}<1 .
$$

Substituting $z=v$ and $z=w$ in (7.10) and taking (7.12) and (7.13) into account, we obtain, in the respective ranges,

$$
v \log y=\frac{u \log y}{1-\alpha_{v}}\left(1+O\left(\frac{1}{\log y}\right)\right)+O(1)
$$

and

$$
w \log y=u \log y\left(1+O_{\varepsilon}\left(R_{\varepsilon}\right)\right)+O(1) .
$$

This implies, in turn,

$$
v \asymp u, \quad|v-u| \ll u\left(\alpha_{v}+1 / \log y\right)
$$

and

$$
w \asymp u, \quad|w-u| \ll_{\varepsilon} u\left(R_{\varepsilon}+1 /(u \log y)\right) .
$$

The desired estimates $(7.6)^{\prime}$ and $(7.8)^{\prime}$ follow from these, noticing that by (6.6) we have

$$
\alpha_{z}^{\prime} \asymp \bar{u} /\left(u^{2} \log y\right)
$$

for any $z$ in the intervals with endpoints $u$ and $v$ or $u$ and $w$. (Actually, this follows directly from (6.6) only if $z \geq 1$, but we have by the above estimates that $z \geq 1+O(1 / \log y)$, and it is easily seen that this does not affect the estimate of $\alpha_{z}^{\prime}$.) We indeed deduce from the last three formulae that

$$
\left|\alpha_{u}-\alpha_{v}\right| \ll \frac{\bar{u}}{u \log y}\left(\alpha_{v}+\frac{1}{\log y}\right) \ll \frac{\alpha_{v}}{\log y}
$$

(since $\left.\alpha_{v} \gg \bar{u} /(u \log y)\right)$ and

$$
\left|\alpha_{u}-\alpha_{w}\right| \ll_{\varepsilon} \frac{\bar{u}}{u \log y}\left(R_{\varepsilon}+\frac{1}{u \log y}\right) \ll R_{\varepsilon}+\frac{1}{u(\log y)^{2}} .
$$

This completes the proof of (2.4).

Next, we establish estimate (2.5) of Theorem 2. By (7.2) we have

$$
\phi_{2}(\alpha, y)=\left(1+O\left(\frac{1}{\log y}\right)\right) \frac{1}{\left(1-y^{-\alpha}\right)^{2}} \int_{1}^{y} \frac{\log t}{t^{\alpha}} d t+O(1)
$$

Estimating the integral by partial summation yields

$$
\begin{aligned}
\int_{1}^{y} \frac{\log t}{t^{\alpha}} d t & =\log y \int_{1}^{y} \frac{d t}{t^{\alpha}}-\int_{1}^{y} \frac{t^{1-\alpha}-1}{t(1-\alpha)} d t \\
& =\log y \int_{1}^{y} \frac{d t}{t^{\alpha}}\left(1+O\left(\frac{1}{(1-\alpha) \log y}\right)\right)
\end{aligned}
$$


Now, if $u \leq \sqrt{y}$, we have, by (7.8),

$$
1-\alpha=1-\frac{\xi(u)}{\log y}+O\left(\frac{1}{(\log y)^{2}}\right) \gg \frac{\log (u+2)}{\log y},
$$

and, if $u>\sqrt{y}, 1-\alpha \gg 1$ by (7.6). Thus

$$
\frac{1}{(1-\alpha) \log y} \ll \frac{1}{\log (u+2)}+\frac{1}{\log y}=E, \quad \text { say, }
$$

and into (7.17) we obtain

$$
\phi_{2}(\alpha, y)=(1+O(E)) \frac{\log y}{\left(1-y^{-\alpha}\right)^{2}} \int_{1}^{y} \frac{d t}{t^{\alpha}}+O(1)
$$

whence by $(7.1)$

$$
\begin{aligned}
\phi_{2}(\alpha, y) & =(1+O(E)) \frac{\left(-\phi_{1}(\alpha, y)\right) \log y}{1-y^{-\alpha}}+O(1) \\
& =(1+O(E)) \frac{\log x \cdot \log y}{1-y^{-\alpha}} .
\end{aligned}
$$

To complete the proof of $(2.5)$, it will therefore be sufficient to show that

$$
\left(1-y^{-\alpha}\right)=\left(1+\frac{\log x}{y}\right)^{-1}\left(1+O\left(\frac{1}{\log y}\right)\right) .
$$

This is clear if $y \geq \log x \cdot \log y$, since (2.4) implies in this circumstance

$$
\alpha \geq \frac{\log \log (1+y)}{\log y}+O\left(\frac{1}{\log y}\right) .
$$

If $y<\log x \cdot \log y$, then hypothesis (7.7) is satisfied. By (7.6) we therefore infer

$$
y^{-\alpha}=\left(1+\frac{y}{\log x}\right)^{-1}\left(1+O\left(\frac{\log (1+y / \log x)}{\log y}\right)\right),
$$

whence

$$
\begin{aligned}
1-y^{-\alpha} & =\left(1+\frac{\log x}{y}\right)^{-1}+O\left(\frac{\log (1+y / \log x)}{(\log y)(1+y / \log x)}\right) \\
& =\left(1+\frac{\log x}{y}\right)^{-1}\left(1+O\left(\frac{(1+(\log x) / y) \log (1+y / \log x)}{(\log y)(1+y / \log x)}\right)\right) .
\end{aligned}
$$

The remainder expression equals

$$
\frac{(\log x) \log (1+y / \log x)}{y \log y}=\frac{1}{\log y} \cdot \frac{\log (1+y / \log x)}{y / \log x} \leq \frac{1}{\log y} .
$$

This completes the proof of (7.18) and hence of (2.5)

Let us now turn to part (ii) of Theorem 2 . We first establish the estimate

$$
\phi_{2}(\alpha, y)=\left(1+O_{\varepsilon}\left(\frac{1}{\log y}\right)\right)(\log y)^{2} \xi^{\prime}(u)^{-1}
$$

for the range (7.9). By (7.13) we have $\alpha \gg_{\varepsilon} 1$, whence by (7.2),

$$
\phi_{2}(\alpha, y)=\left(1+O_{\varepsilon}\left(\frac{1}{\log y}\right)\right) \int_{1}^{y} \frac{\log t}{t^{\alpha}} d t
$$


Moreover, (7.8) shows that replacing $\alpha$ in the integral by $\alpha_{w}=1-\xi(u) / \log y$ introduces at most an error factor of $1+O_{\varepsilon}(1 / \log y)$. The modified integral can be easily evaluated. We have

$$
\begin{aligned}
\int_{1}^{y} \frac{\log t}{t^{\alpha_{w}}} d t & =-\left[\frac{d}{d \sigma} \int_{1}^{y} \frac{d t}{t^{\sigma}}\right]_{\sigma=\alpha_{w}}=\frac{d}{d u}\left(\frac{y^{1-\alpha_{w}}-1}{1-\alpha_{w}}\right) \cdot\left(-\frac{d \alpha_{w}}{d u}\right)^{-1} \\
& =\frac{d}{d u}\left(\frac{e^{\xi(u)}-1}{\xi(u)} \cdot \log y\right) \frac{\log y}{\xi^{\prime}(u)}=(\log y)^{2} \xi^{\prime}(u)^{-1}
\end{aligned}
$$

This yields (7.19).

The proof of (2.6) now follows in a rather simple way. From (7.8) and (7.19) we readily get

$$
\left(\alpha \sqrt{2 \pi \phi_{2}(\alpha, y)}\right)^{-1}=\left(\frac{\xi^{\prime}(u)}{2 \pi}\right)^{1 / 2} \frac{1}{\log y} \exp \left\{O_{\varepsilon}\left(\frac{\log (u+1)}{\log y}\right)\right\} .
$$

Moreover, we also have by (7.8) that

$$
x^{\alpha}=x \exp \left\{-u \xi(u)+O_{\varepsilon}\left(u R_{\varepsilon}+\frac{1}{\log y}\right)\right\} .
$$

Finally, we may write

$$
\varsigma(\alpha, y)=\varsigma(1, y) \exp \left\{-\int_{\alpha}^{1} \phi_{1}(\sigma, y) d \sigma\right\}
$$

where, by Mertens' Theorem

$$
\varsigma(1, y)=e^{\gamma} \log y(1+O(1 / \log y))
$$

and, by Lemma 13 ,

$$
\begin{aligned}
-\int_{\alpha}^{1} \phi_{1}(\sigma, y) d \sigma & =\left(1+O_{\varepsilon}\left(R_{\varepsilon}\right)\right) \int_{\alpha}^{1}\left(\frac{y^{1-\sigma}-1}{1-\sigma}\right) d \sigma+O(1-\alpha) \\
& =\left(1+O_{\varepsilon}\left(R_{\varepsilon}\right)\right) \int_{0}^{(1-\alpha) \log y} \frac{e^{s}-1}{s} d s+O(1-\alpha)
\end{aligned}
$$

with an obvious interpretation in the case $\alpha \geq 1$. Using (7.8), the last expression becomes

$$
\int_{0}^{\xi(u)} \frac{e^{s}-1}{s} d s+O_{\varepsilon}\left(\frac{\log (u+1)}{\log y}+u R_{\varepsilon}\right) .
$$

The desired formula (2.6) now follows on collecting these estimates.

This completes the proof of Theorem 2 .

\section{REFERENCES}

1. K. Alladi, The Turán-Kubilius inequality for integers without large prime factor, J. Reine Angew Math. 335 (1982), 180-196.

2. N. G. de Bruijn, The asymptotic behaviour of a function occurring in the theory of primes, J. Indian Math. Soc. (N.S.) 15 (1951), 25-32.

3. Akad. Wetensch. Proc. Ser. A 54 (1951), 50-60.

4. __ On the number of positive integers $\leq x$ and free of prime factors $>y$. II, Nederl. Akad. Wetensch. Proc. Ser. A 69 (1966), 239-247 = Indag. Math. 28 (1966), 239-247. 
5. E. R. Canfield, On the asymptotic behaviour of the Dickman-de Bruijn function, Congr. Numer. 38 (1982), 139-148.

6. E. R. Canfield, P. Erdös and C. Pomerance, On a problem of Oppenheim concerning "Factorisatio Numerorum", J. Number Theory 17 (1983), 1-28.

7. K. Dickman, On the frequency of numbers containing prime factors of a certain relative magnitude, Ark. Mat. Astr. Fys. 22 (1930), 1-14.

8. W. J. Ellison and M. Mendes-France, Les nombres premiers, Hermann, Paris, 1975.

9. V. Ennola, On numbers with small prime divisors, Ann. Acad. Sci. Fenn. Ser. A I 440 (1969), 16pp.

10. D. Hensley, the number of positive integers $\leq x$ and free of prime divisors $>y$, J. Number Theory 21 (1985), 286-298.

11. _ A property of the counting function of integers with no large prime factors, J. Number Theory 22 (1986), 46-74.

12. A. Hildebrand, On the number of positive integers $\leq x$ and free of prime factors $>y$, J. Number Theory 22 (1986), 289-307.

13.

14. H. Maier, On integers free of large prime divisors, Preprint.

15. K. Norton, Numbers with small prime factors and the least kth power non-residue, Mem. Amer. Math. Soc. No. 106 (1971).

16. K. Prachar, Primzahlverteilung, Springer, Berlin, 1957.

17. R. Rankin, The difference between consecutive prime numbers, J. London Math. Soc. 13 (1938), 242-247.

18. A. I. Vinogradov, On numbers with small prime divisors, Dokl. Akad. Nauk SSSR (N.S.) 109 (1956), 683-686. (Russian)

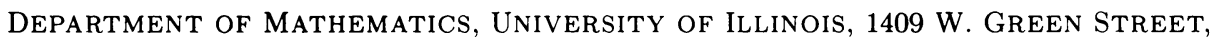
URBANA, ILLINOIS 61801

Département de Mathématiques, Université De NANCY I, B. P. 239, 54506 VanDOEUVRE CEDEX, FRANCE 